\title{
ON NEARLY ABSOLUTELY ISOLATED HYPERSURFACE SINGULARITIES OF DIMENSION 2
}

\author{
JIN-GEN YANG
}

\begin{abstract}
A formula for the geometric genus of a nearly absolutely isolated hypersurface singularitiy of dimension 2 is found by using the canonical resolution. An upper bound for the fundamental cycle of such singularity is also given.
\end{abstract}

Introduction. Let $\pi: M \rightarrow V$ be a resolution of an isolated singularity of dimension 2. The number $\operatorname{dim} H^{0}\left(V, R^{1} \pi_{*}\left(\mathcal{O}_{M}\right)\right)$ is defined to be the geometric genus of the singularity. We study the case in which $V$ can be embeded in $\mathbf{C}^{3}$. Our major result is a formula (Theorem 2) for the geometric genus when the singularity is nearly absolutely isolated (see $\$ 2$ for definition). Our proof is based on the canonical resolution of an $m$-tuple point, which is developed in the first section. The case $m=2$ is well-known (cf. [2, pp. 47-48]). Finally we give a bound for the fundamental cycle of that kind of singularity as well as for its self-intersection number (Theorem 3).

The base field is the complex number field C. A singular point $p$ always means a hypersurface point of dimension 2 . Sometimes we use the same notation for a line bundle and its corresponding divisor if it will not cause confusion.

1. $m$-tuple covering. Let $m \geqslant 2$. Let $Y$ be a smooth surface covered by affine open sets $\left\{U_{i}\right\}_{i \in I}$. Let $C_{0}, \ldots, C_{m-2}$ be effective divisors on $Y$ locally defined in $U_{i}$ by equations $c_{s, i}=0(0 \leqslant s \leqslant m-2, i \in I)$. Suppose there is a line bundle $F$ over $Y$ with transition function $\left\{f_{i j}\right\}$ over $\left\{U_{i} \cap U_{j}\right\}$ such that

$$
c_{s, i}=f_{i j}^{m-s} c_{s, j}
$$

for all $0 \leqslant s \leqslant m-2$. Let $\phi_{i}$ be the fibre coordinates over $U_{i}$. Then the equations

$$
\phi_{i}^{m}+c_{m-2, i} \phi_{i}^{m-2}+\cdots+c_{0, i}=0
$$

give rise to a surface $X$ in $F$ and the projection map from $F$ to $Y$ induces a finite morphism $f: X \rightarrow Y$ of degree $m$.

Definition. The surface $X$ constructed as above is called the $m$-tuple cover of $Y$ with branch locus data $\left(C_{0}, \ldots, C_{m-2}\right)$.

Let $D_{i}$ be the discriminant of the equation (2) for $i \in I$. Then $\left\{D_{i}\right\}_{i \in I}$ give rise to a divisor on $Y$, denoted by $D$. Obviously $D$ is the branch locus of the map $f$. The map $f$ is called totally ramified at a point $p \in Y$ if $f^{-1}(p)$ consists of one point.

Received by the editors September 12, 1985.

1980 Mathematics Subject Classification (1985 Revision). Primary $14 J 17$. 
Since the degree $(m-1)$ term in (2) has zero coefficient, the set of all totally ramified points in $Y$ is $C_{0} \cap \cdots \cap C_{m-2}$.

For any point $p \in Y$, let $\mu_{p}\left(C_{s}\right)$ be the multiplicity of $C_{s}$ at $p$. Then a point $p \in C_{0} \cap \cdots \cap C_{m-2}$ gives rise to an $m$-tuple point on $X$ if and only if $\mu_{p}\left(C_{s}\right) \geqslant m$ $-s$ for $0 \leqslant s \leqslant m-2$.

Suppose $x=f^{-1}(p)$ is an isolated multiple point of $X$. We call $x$ a simple $m$-tuple point if there is an open neighborhood $U$ of $p$ such that the support of the divisor $C_{0}+C_{1}+\cdots+C_{m-2}$ in $U$ has two irreducible components which are nonsingular at the point $p$ with normal crossing.

Suppose that $X$ has only a finite number of $m$-tuple points. Let $p \in C_{0}$ $\cap \cdots \cap C_{m-2}$ be such that $f^{-1}(p)$ is an $m$-tuple point. Let $q_{1}: Y_{1} \rightarrow Y$ be the blowing-up of $Y$ at $p$. Let $E_{1}=q_{1}^{-1}(p)$. Let

$$
r_{1}=\min \left(\left[\mu_{p}\left(C_{m-2}\right) / 2\right], \ldots,\left[\mu_{p}\left(C_{0}\right) / m\right]\right) \text {, }
$$

where $[a]$ is the integer part of $a$. We call $r_{1}$ the multiplicity of the branch locus data $\left(C_{0}, \ldots, C_{m-2}\right)$ at $p$. Let the divisors $C_{1, s}(0 \leqslant s \leqslant m-2)$ be defined by

$$
C_{1, s}=q_{1}^{*}\left(C_{s}\right)-r_{1}(m-s) E_{1} \text {. }
$$

Let $F_{1}=q_{1}^{*}(F)-r_{1} E_{1}$. It is easy to check that the line bundle $F_{1}$ and the divisors $C_{1,0}, \ldots, C_{1, m-2}$ on $Y_{1}$ satisfy the condition (1). Let $f_{1}: X_{1} \rightarrow Y_{1}$ be the $m$-tuple cover of $Y_{1}$ with branch locus data $\left(C_{1,0}, \ldots, C_{1, m-2}\right)$. Obviously there is a map $\pi_{1}$ : $X_{1} \rightarrow X$ such that the diagram

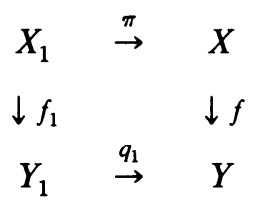

commutes.

By the choice of $r_{1}$, we see that $X_{1}$ has only a finite number of $m$-tuple points. We can repeat the same procedure for any $m$-tuple point on $X_{1}$.

LEMMA. The above procedure will terminate after a finite number of steps.

Proof. It is easy to see that after a finite number of steps there are at most finitely many simple $m$-tuple points on the top surface. Thus we may assume that $f^{-1}(p)$ is a simple $m$-tuple point.

By definition, the simple $m$-tuple point $f^{-1}(p)$ is analytically isomorphic to an $m$-tuple point given by

$$
x^{m}+y^{s_{m-2}} z^{t_{m-2}} x^{m-2}+\cdots+y^{s_{1}} z^{t_{1}} x+y^{s_{0}} z^{t_{0}}=0
$$

satisfying
(A) $s_{i}+t_{i} \geqslant m-i$ for $0 \leqslant i \leqslant m-2$;
(B) $\min _{0 \leqslant i \leqslant m-2}\left(s_{i}+i\right)<m$;
(C) $\min _{0 \leqslant i \leqslant m-2}\left(t_{i}+i\right)<m$.
In particular, $\mu_{p}\left(C_{i}\right)=s_{i}+t_{i}$ for $0 \leqslant i \leqslant m-2$. 
Let $S=\left\{0 \leqslant i \leqslant m-2: s_{i} /(m-i)=\min _{0 \leqslant k \leqslant m-2}\left(s_{k} /(m-k)\right)\right\}$ and

$$
T=\left\{0 \leqslant i \leqslant m-2: t_{i} /(m-i)=\min _{0 \leqslant k \leqslant m-2}\left(t_{k} /(m-k)\right)\right\} .
$$

Let $\alpha=\min _{i \in T}\left\{s_{i} /(m-i)\right\}+\min _{i \in S}\left\{t_{i} /(m-i)\right\}$. We call this rational number $\alpha$ the "grade" of the $m$-tuple point $f^{-1}(p)$.

The exceptional divisor $E_{1}$ of the blowing-up $q_{1}$ intersects the branch locus of $f_{1}$ at two distinct points $p_{1}$ and $p_{1}^{\prime}$, which are the only points on $Y_{1}$ that might give rise to $m$-tuple points on $X_{1}$. Since the discussions of $p_{1}$ and $p_{1}^{\prime}$ are the same, it suffices to consider $p_{1}$. If $f_{1}^{-1}\left(p_{1}\right)$ is not an $m$-tuple point then there is nothing to prove. So we assume $f_{1}^{-1}\left(p_{1}\right)$ is an $m$-tuple point. Then it can be represented by

$$
x^{m}+y^{s_{m-2}} z^{s_{m-2}+t_{m-2}-2 r_{1}} x^{m-2}+\cdots+y^{s_{1}} z^{s_{1}+t_{1}-(m-1) r_{1}} x+y^{s_{0}} z^{s_{0}+t_{0}-m r_{1}}=0
$$

where $r_{1}=\min \left(\left[\left(s_{m-2}+t_{m-2}\right) / 2\right], \ldots,\left[\left(s_{0}+t_{0}\right) / m\right]\right)$. We want to show that the "grade" $\alpha_{1}$ of $f_{1}^{-1}\left(p_{1}\right)$ is smaller than $\alpha$. Assume that $j \in T$ and $k \in S$ are such that $s_{j} /(m-j)=\min _{i \in T}\left\{s_{i} /(m-i)\right\}$ and $t_{k} /(m-k)=\min _{i \in S}\left\{t_{i} /(m-i)\right\}$. We also assume that $\left(s_{n}+t_{n}\right) /(m-n)=\min _{0 \leqslant i \leqslant m-2}\left\{\left(s_{i}+t_{i}\right) /(m-i)\right\}$. By definition,

$$
\boldsymbol{\alpha}_{1}=\min _{i \in T_{1}}\left\{s_{i} /(m-i)\right\}+\min _{i \in S_{1}}\left\{\left(s_{i}+t_{i}-(m-i) r_{1}\right) /(m-i)\right\}
$$

where $S_{1}=S$ and

$$
\begin{aligned}
T_{1}=\{0 \leqslant i \leqslant m-2:( & \left.s_{i}+t_{i}-(m-i) r_{1}\right) /(m-i) \\
& \left.=\min _{0 \leqslant k \leqslant m-2}\left(\left(s_{k}+t_{k}-(m-k) r_{1}\right) /(m-k)\right)\right\} .
\end{aligned}
$$

Clearly $n \in T_{1}$. Hence $\alpha_{1} \leqslant s_{n} /(m-n)+\left(s_{k}+t_{k}-(m-k) r_{1}\right) /(m-k)$. Since $t_{n} /(m-n) \geqslant t_{j} /(m-j)$ and $\left(s_{n}+t_{n}\right) /(m-n) \leqslant\left(s_{j}+t_{j}\right) /(m-j)$ we have $s_{n} /(m-n) \leqslant s_{j} /(m-j)$. On the other hand, $s_{k} /(m-k)<1$ (by property (B)) and $r_{1} \geqslant 1$ imply that $\left(s_{k}+t_{k}-(m-k) r_{1}\right) /(m-k)<t_{k} /(m-k)$. Hence

$$
\begin{aligned}
\alpha_{1} & \leqslant s_{j} /(m-j)+\left(s_{k}+t_{k}-(m-k) r_{1}\right) /(m-k) \\
& <s_{j} /(m-j)+t_{k} /(m-k)=\alpha .
\end{aligned}
$$

Since all "grades" during the process of the resolution are nonnegative rational numbers with denominators less than a fixed number $m$ !, the procedure must terminate after a finite number of steps.

If $X$ has only a finite number of $m$-tuple points then, by the lemma, there will be a commutative diagram

$$
\begin{array}{cccccccccc}
X_{n} & \stackrel{\pi_{n}}{\rightarrow} & X_{n-1} & \rightarrow \cdots & X_{2} & \stackrel{\pi_{2}}{\rightarrow} & X_{1} & \stackrel{\pi_{1}}{\rightarrow} & X \\
f_{n} \downarrow & & f_{n-1} \downarrow & & & f_{2} \downarrow & & f_{1} \downarrow & & f \downarrow \\
Y_{n} & \stackrel{q_{n}}{\rightarrow} & Y_{n+1} & \rightarrow \cdots & \rightarrow & Y_{2} & \stackrel{q_{2}}{\rightarrow} & Y_{1} & \stackrel{q_{1}}{\rightarrow} & Y
\end{array}
$$

such that $X_{n}$ has no $m$-tuple points. But $X_{n}$ is not necessarily normal.

DEFINITION. The surface $X_{n}$ is called the canonical resolution of $m$-tuple points in $X$. If $X_{n}$ is normal, then the above resolution is called a normal canonical resolution. 
Here the term "resolution" is not in the usual sense. Note that during the canonical resolution each map $q_{i}$ is related to a number $r_{i}$, which is the multiplicity of the branch locus data of the $m$-tuple covering map $f_{i-1}$. The sequence $r_{1}, \ldots, r_{n}$ can be used to decide some geometric invariants of the surface $X_{n}$.

THEOREM 1. The holomorphic Euler characteristic of the canonical resolution $X_{n}$ of the $m$-tuple point $p$ in $X$ is given by

$$
\chi\left(X_{n}, \mathcal{O}_{X_{n}}\right)=m \chi\left(Y, \mathcal{O}_{Y}\right)-\sum_{i=1}^{n}\left(\frac{(m-1) m(2 m-1)}{12} r_{i}^{2}-\frac{(m-1) m}{4} r_{i}\right) .
$$

Proof. Let $F_{i}^{\#}$ be the completion of the line bundle $F_{i}$. Let $\left[Y_{i}\right]$ be the zero section of $F_{i}^{\#}$. There are exact sequences

$$
\begin{aligned}
0 & \rightarrow \mathcal{O}_{F_{i}^{*}}\left(-X_{i}\right) \rightarrow \mathcal{O}_{F_{i}^{*}} \rightarrow \mathcal{O}_{X_{i}} \rightarrow 0, \\
0 & \rightarrow \mathcal{O}_{F_{i}^{*}}\left(-\left[Y_{i}\right]\right) \rightarrow \mathcal{O}_{F_{i}^{*}} \rightarrow \mathcal{O}_{Y_{i}} \rightarrow 0, \\
0 & \rightarrow \mathcal{O}_{F_{i}^{*}}\left(-2\left[Y_{i}\right]\right) \rightarrow \mathcal{O}_{F_{i}^{*}}\left(-\left[Y_{i}\right]\right) \rightarrow \mathcal{O}_{Y_{i}}\left(-F_{i}\right) \rightarrow 0, \\
\quad & \vdots \\
0 & \rightarrow \mathcal{O}_{F_{i}^{*}}\left(-X_{i}\right) \rightarrow \mathcal{O}_{F_{i}^{*}}\left(-(m-1)\left[Y_{i}\right]\right) \rightarrow \mathcal{O}_{Y_{i}}\left(-(m-1) F_{i}\right) \rightarrow 0
\end{aligned}
$$

due to the facts $m\left[Y_{i}\right] \sim X_{i}$ in $F_{i}^{\#}$ and $\left.Y_{i}\right|_{Y_{i}} \sim F_{i}$.

Using these exact sequences and the Riemann-Roch Theorem, we have

$$
\begin{aligned}
\chi\left(X_{i}, \mathcal{O}_{X_{i}}\right)=\chi\left(F_{i}^{\#}, \mathcal{O}_{F_{i}^{*}}\right)-\chi\left(F_{i}^{\#}, \mathcal{O}_{F_{i}^{*}}\left(-X_{i}\right)\right) \\
=\chi\left(F_{i}^{\#}, \mathcal{O}_{F_{i}^{*}}\right)-\chi\left(F_{i}^{\#}, \mathcal{O}_{F_{i}^{*}}\left(-(m-1)\left[Y_{i}\right]\right)\right)+\chi\left(Y_{i}, \mathcal{O}_{Y_{i}}\left(-(m-1) F_{i}\right)\right) \\
=\cdots \\
=\chi\left(Y_{i}, \mathcal{O}_{Y_{i}}\right)+\chi\left(Y_{i}, \mathcal{O}_{Y_{i}}\left(-F_{i}\right)\right)+\cdots+\chi\left(Y_{i}, \mathcal{O}_{Y_{i}}\left(-(m-1) F_{i}\right)\right) \\
=m \chi\left(Y_{i}, \mathcal{O}_{Y_{i}}\right)+\left(\frac{1}{2}\right) F_{i}\left(F_{i}+k_{i}\right)+\cdots+\left(\frac{1}{2}\right)(m-1) F_{i}\left((m-1) F_{i}+k_{i}\right) \\
=m \chi\left(Y_{i}, \mathcal{O}_{Y_{i}}\right)+\left(\frac{1}{2}\right)\left(\sum_{r=1}^{m-1} r^{2}\right) F_{i}^{2}+((m-1) m / 4) F_{i} k_{i} \\
=m \chi\left(Y, \mathcal{O}_{Y}\right)+((m-1) m(2 m-1) / 12) F_{i}^{2}+((m-1) m / 4) F_{i} k_{i}
\end{aligned}
$$

where $k_{i}$ is the canonical divisor of $Y_{i}$. Since $F_{i}=q_{i}^{*}\left(F_{i-1}\right)-r_{i} E_{i}$ and $k_{i}=$ $q_{i}^{*}\left(k_{i-1}\right)+E_{i}$, the theorem follows immediately.

2. Nearly absolutely isolated $m$-tuple points. Let $p$ be an isolated $m$-tuple surface singularity which can be embedded in $\mathbf{C}^{3}$. In other words, $p \in V \subset \mathbf{C}^{3}$ where $V$ is a surface. We may assume that $p$ is the only singularity on $V$. Let $\pi: M \rightarrow V$ be a resolution of $p$. The set $A=\pi^{-1}(p)$ is called the exceptional set. The number $h(p)=\operatorname{dim} H^{0}\left(V, R^{1} \pi_{*}\left(\mathcal{O}_{M}\right)\right)$ is called the geometric genus of $p$.

In this section we use the construction of $m$-tuple covering and Theorem 1 to find a formula for $h(p)$ for a certain type of $p$.

Denote by $x, y, z$ the coordinates of $\mathbf{C}^{3}$. Assume that $p=(0,0,0)$. Since $V \subset \mathbf{C}^{3}$, $V$ is defined by a single equation. Without loss of generality, we may assume the equation is given by

$$
z^{m}=a_{m-2}(x, y) z^{m-2}+a_{m-3}(x, y) z^{m-3}+\cdots+a_{1}(x, y) z+a_{0}(x, y)
$$


where $a_{0}(x, y), \ldots, a_{m-2}(x, y)$ are polynomials in $x, y$. For any polynomial $g(x, y)$, define the order of $g(x, y)$ to be the lowest degree of all its terms, denoted by $\operatorname{ord}(g)$. Since $p$ has multiplicity $m$, ord $\left(a_{i}\right) \geqslant m-i$. Let $x, y, w$ be the homogeneous coordinates in $\mathbf{P}^{2}$. Let

$$
N=\max _{0 \leqslant i \leqslant m-2}\left(\left[\frac{\operatorname{deg}\left(a_{i}\right)+m-i-1}{m-i}\right]\right)
$$

and let $A_{i}(x, y, w)=w^{N(m-i)} a_{i}(x / w, y / w)$ for each $0 \leqslant i \leqslant m-2$. Each $A_{i}(x, y, w)$ is a homogeneous polynomial of degree $(m-i) N$. Let $F$ be the line bundle $\mathcal{O}_{\mathbf{P}^{2}}(N)$. Let $C_{i}$ be the zero locus of $A_{i}(x, y, w)$ on $\mathbf{P}^{2}$. Then it is easy to see that the fibre coordinates of $F$ satisfy (1) for each affine open subset of $\mathbf{P}^{2}$. Let $X$ be the $m$-tuple cover of $\mathbf{P}^{2}$ with branch locus data $\left(C_{0}, \ldots, C_{m-2}\right)$ as defined before. Then $V$ is merely a subscheme of $X$. Hence we may think of $X$ as our original $V$ and $p \in X$ sits over $0 \in \mathbf{P}^{2}$. Let $\pi: X_{n} \rightarrow X$ be the canonical resolution of the $m$-tuple point $p$ as defined in the previous section. Suppose $X_{n}$ is normal. Then there are finite number of multiple points of $X_{n}$ on $\pi^{-1}(p)$ with multiplicities smaller than $m$. These are called infinitely near multiple points of $p$. Suppose that their canonical resolutions are normal. Then we can consider their infinitely near multiple points and repeat the same process. If all canonical resolutions involved in the above process are normal then the $m$-tuple point $p$ is called nearly absolutely isolated. (Remember that an isolated singularity is absolutely isolated if it can be resolved by a sequence of blowing-ups.) Obviously all isolated double points are nearly absolutely isolated.

THEOREM 2. Let $p$ be a nearly absolutely isolated m-tuple point of dimension 2 which can be embedded in $\mathbf{C}^{3}$. Then the geometric genus $h(p)$ of $p$ is given by the formula

$$
h(p)=\sum_{\lambda=2}^{m} \sum_{q \in I_{\lambda}} \sum_{i=1}^{n_{\lambda . q}}\left(\frac{(\lambda-1) \lambda(2 \lambda-1)}{12} r_{i, q}^{2}-\frac{(\lambda-1) \lambda}{4} r_{i, q}\right)
$$

where $I_{\lambda}$ is the set of all infinitely near $\lambda$-tuple points of $p$ and $r_{1, q}, \ldots, r_{n_{\lambda, q}, q}$ is the sequence of multiplicities of the branch locus data of $q$.

Proof. Let $\tilde{X}$ be the resolution of $p$ in the sense that there is a morphism $\xi$ : $\tilde{X} \rightarrow X$ such that $\left.\xi\right|_{\tilde{X}-\xi^{-1}(p)}$ is an isomorphism and $\tilde{X}$ is nonsingular on $\xi^{-1}(p)$. Theorem 1 implies that $\chi\left(X, \mathcal{O}_{X}\right)-\chi\left(\tilde{X}, \mathcal{O}_{\tilde{X}}\right)$ is equal to the right-hand side of (4). It is well known that $\chi\left(\tilde{X}, \mathcal{O}_{\tilde{X}}\right)=\chi\left(X, \mathcal{O}_{X}\right)-h(p)$. Therefore (4) is true.

Since $p$ has multiplicity $m$, ord $\left(a_{i}(x, y)\right) \geqslant m-i$. Let $T_{i}$ be the tangent cone of $C_{i}$ at 0 for $2 \leqslant i \leqslant m-2$. We say that $p$ is generic when 0 is an ordinary $(m-i)$-tuple point of $C_{i}$ for each $i$ and $T_{i} \cap T_{j}=0$ for any $i \neq j$.

COROLlary. If $p$ is a generic m-tuple point, then $h(p)=\left(\begin{array}{c}m \\ 3\end{array}\right)$.

Proof. Being generic implies that $p$ is resolved by blowing up once. Therefore in (4) there is only one term

$$
\frac{(m-1) m(2 m-1)}{12}-\frac{(m-1) m}{4}=\left(\begin{array}{c}
m \\
3
\end{array}\right) .
$$


3. Fundamental cycles. On the exceptional set $A$ there is a unique positive cycle $Z$ satisfying

(i) $A_{i} Z \leqslant 0$ for any irreducible component $A_{i}$ of $Z$;

(ii) $Z$ is minimal with respect to the above property.

The cycle $Z$ is called the fundamental cycle. For details, see [1].

Let $p$ be a nearly absolutely isolated $m$-tuple point as in the last section. There is a commutative diagram

$$
\begin{array}{rccccccccccc}
\tilde{X} \stackrel{\pi_{n}}{\rightarrow} & X_{n} & \stackrel{\pi_{n}}{\rightarrow} & X_{n-1} & \stackrel{\pi_{n-1}}{\rightarrow} & \cdots & \rightarrow & X_{2} & \stackrel{\pi_{2}}{\rightarrow} & X_{1} & \stackrel{\pi_{1}}{\rightarrow} & X \\
& f_{n} \downarrow & & f_{n-1} \downarrow & & & & & & & & \\
& Y_{n} & \overrightarrow{q_{n}} & Y_{n-1} & \underset{q_{n-1}}{\rightarrow} & \cdots & \rightarrow & Y_{2} & \overrightarrow{q_{2}} & Y_{1} & \overrightarrow{q_{1}} & Y
\end{array}
$$

where $\psi$ is the minimal resolution of $X_{n}$. Let $\pi=\pi_{1} \circ \pi_{2} \circ \cdots \circ \pi_{n} \circ \psi$ and let $\sigma=f_{1} \circ \pi_{2} \circ \cdots \circ \pi_{n} \circ \psi$. Then $\pi: \tilde{X} \rightarrow X$ is a resolution of $p$, though not necessarily minimal.

THEOREM 3. Let $Z$ be the fundamental cycle of $p$ on $\tilde{X}$. Then $Z \leqslant \sigma^{*}\left(E_{1}\right)$, the pull-back of the exceptional divisor $E_{1}=q_{1}^{-1}(0)$ on $Y_{1}$. Moreover, $Z^{2} \geqslant-m$ and the equality holds if and only if $Z=\sigma^{*}\left(E_{1}\right)$.

Proof. Let $\bar{Z}=\sigma^{*}\left(E_{1}\right)$. Then $\bar{Z}^{2}=m E_{1}^{2}=-m$. For any irreducible component $A_{i}$ of $A$, we have

$$
A_{i} \bar{Z}=\left(\sigma_{*} A_{i}\right) E_{1}= \begin{cases}0, & \text { if } \sigma\left(A_{i}\right) \text { is a point; } \\ -1, & \text { if } \sigma\left(A_{i}\right)=E_{1} .\end{cases}
$$

Hence $\bar{Z}$ satisfies the first condition of the definition of fundamental cycle. Therefore $Z \leqslant \bar{Z}$.

Write $\bar{Z}=Z+Z^{\prime}$. Then $Z^{2}+2 Z Z^{\prime}+Z^{\prime 2}=-m$. By the definition of $Z, Z Z^{\prime}$ $\leqslant 0$. Since the intersection matrix of $A$ is negative definite, $Z^{\prime 2} \leqslant 0$ and $Z^{\prime 2}=0$ if and only if $Z^{\prime}=0$. This finishes the proof.

COROLlaRY. If $p$ is generic, then $Z=\sigma^{*}\left(E_{1}\right)$ and $Z^{2}=-m$.

Proof. In this case $\tilde{X}=X_{1}$, and $\sigma^{*}\left(E_{1}\right)$ has no multiple components. Hence $Z=\sigma^{*}\left(E_{1}\right)$.

\section{REFERENCES}

1. M. Artin, Some numerical criteria for contractibility of curves on an algebraic surface, Amer. J. Math. 84 (1962), 485-496.

2. E. Horikawa, On deformations of quintic surfaces, Invent. Math. 31 (1975), 43-85.

3. D. Kirby, The structure of an isolated multiple point of a surface. I, Proc. London Math. Soc. 6 (1956), 597-609; II, 7 (1957), 1-28.

Department of Mathematics, University of TeXas, Austin, TeXas 78712 